\title{
Fuzzy Inference System based Contrast Enhancement
}

\author{
Balasubramaniam Jayaram, ${ }^{1}$ Kakarla V.V.D.L. Narayana, ${ }^{2}$ V. Vetrivel $^{2}$ \\ ${ }^{1}$ Department of Mathematics, Indian Institute of Technology Hyderabad, Yeddumailaram - 502 205, INDIA \\ ${ }^{2}$ Department of Mathematics, Indian Institute of Technology Madras, Chennai - 600 036, INDIA
}

\begin{abstract}
In this work, we propose a fuzzy inference system based contrast enhancement of gray level images. We propose a new method of generating the fuzzy if-then rules specific to a given image based on the local information available to be used by a fuzzy inference system. To this end, we only generate a partial histogram and not a complete histogram thus saving on computational costs. We also give a compartive study of our approach and some classical and existing fuzzy techniques, and show that the enhanced images from the proposed algorithm are comparable.
\end{abstract}

Keywords: Image Enhancement, Contrast Stretching, Fuzzy Inference Systems, Fuzzy Partition, Histogram Equalisation, Histogram Matching, Gray level transformations.

\section{Image enhancement}

Enhancement is a fundamental task in digital image processing and analysis. Image enhancement seeks to improve the visual representation of an image to enhance its interpretability by either a human or a machine. Image enhancement encompasses a variety of operations such as noise removal, deblurring, gray-level dynamic range modification. Contrast enhancement is among them and is often part of image processing systems in the pre-processing and/or post-processing stage.

The principal objective of enhancement is to process an image so that the result is more suitable than the original image for a specific application. The word specific is more important, because a method that is quite useful for enhancing X-ray images may not necessarily be the best approach for enhancing natural sceneries. When an image is processed for visual interpretation, the viewer becomes the ultimate judge of how well a method works.

\subsection{Spatial Domain Methods}

The term spatial domain refers to the set of all pixels composing an image. Spatial domain methods are procedures that operate directly on these pixels. If $f(x, y)$ denotes the input image and $g(x, y)$ the processed image, the different spatial domain procedures can be seen as an operator $T$ on $f$ such that

$$
g(x, y)=T\left[f\left(x^{\prime}, y^{\prime}\right)\right], \quad\left(x^{\prime}, y^{\prime}\right) \in \mathcal{N}(x, y),
$$

where $\mathcal{N}(x, y)$ is some neigbhourhood of the point $(x, y)$. Usually, a neighborhood about a point $(x, y)$ is taken to be the square or rectangular subimage area centered at $(x, y)$. The center of the subimage is moved from pixel to pixel starting, say, at the top left corner. The operator $T$ now refers to applying some transformation at each location $\left(x^{\prime}, y^{\prime}\right) \in \mathcal{N}(x, y)$ and suitably aggregating to yield the output $g$, at that location. The process utilizes only the pixels in the area of the image spanned by the neighborhood.

\subsection{Contrast Stretching}

From the above, we see that any continuous function with domain as the grey values of the input image and the range as the grey levels of the output image can be used dependent on the applications / utility. Often the images to be processed suffer from low contrasts. Low contrast images can result from poor illumination, lack of dynamic range in the imaging sensor, or even wrong setting of a lens aperture during image acquisition. Hence, there is a need for contrast-stretching, i.e., highlighting a specific range of gray levels in an image. The idea behind contrast stretching is to increase the dynamic range of the gray levels in the image being processed. Applications include enhancing features such as masses of water in satellite imagery and enhancing flaws in X-ray images. This is generally achieved in one of the following two ways: (a) To display a high value for all gray levels in the range of interest and a low value for all other gray levels (b) Brighten the desired range of gray levels but preserve the background and gray-level tonalities in the image. In essence, we stretch the contrast present in a given image.

\subsection{Histogram Processing}

Histograms are the basis for numerous spatial domain processing techniques. Histogram manipulation can be used effectively for image enhancement. Histograms are simple to calculate and also lend themselves to economic hardware implementations, thus making them a popular tool for real-time image processing. 
The histogram of a digital image with gray levels in the range $[0, L-1]$ is a discrete function $h\left(r_{k}\right)=n_{k}$, where $r_{k}$ is the $k^{t h}$ gray level and $n_{k}$ is the number of pixels in the image having gray level $r_{k}$. It is common practice to normalize a histogram by dividing each of its values by total number of pixels in the image, denoted by $n$. Thus a normalized histogram is given by $p\left(r_{k}\right)=\frac{n_{k}}{n}$, for $k=0,1, \ldots ., L-1$. Loosely speaking, $p\left(r_{k}\right)$ gives an estimate of the probability of occurrence of gray level $r_{k}$. Note that the sum of all components of a normalized histogram is equal to 1 .

\subsection{Histogram Equalization}

Histogram equalization is an approach to enhance a given image. The approach is to design a transformation $T($.$) such that the gray values in the output$ are uniformly distributed in $[0,1]$. Consider a discrete formulation and allow pixel values to be in the interval $[0, L-1]$. We need to design a gray value transformation $s=T(r)$, based on the histogram of the input image, which will enhance the image.

The probability of occurrence of gray level $r_{k}$ in an image is approximated by

$$
p_{r}\left(r_{k}\right)=\frac{n_{k}}{n} \quad k=0,1,2, \ldots, L-1,
$$

where, as noted in the beginning of this section, $n$ is the total number of pixels in the image, $n_{k}$ is the number of pixels that have gray levels $r_{k}$, and $L$ is the total number of possible gray levels in the image. The transformed gray levels are obtained as follows: For $k=0,1,2, \ldots, L-1$,

$$
s_{k}=T\left(r_{k}\right)=\sum_{j=0}^{k} p_{r}\left(r_{j}\right)=\sum_{j=0}^{k} \frac{n_{j}}{n} .
$$

Thus, a processed image is obtained by mapping each pixel with level $r_{k}$ in the input image into a corresponding pixel with level $s_{k}$ in the output image via (3). As indicated earlier, a plot of $p_{r}\left(r_{k}\right)$ versus $r_{k}$ is called the histogram of the image. The transformation given in (3) is called histogram equalization or histogram linearization, since this transformation has the tendency of spreading the histogram of the input image so that the levels of the histogram-equalized image will span the full range of the gray scale. In addition the method is also fully "automatic", i.e., given an image, the process of histogram equalization consists simply of implementing (3), which is based on information that can be extracted directly from the given image.

\subsection{Main Focus of this work}

In this paper, we propose a fuzzy inference system based contrast enhancement of gray level images. We present a new method of generating the fuzzy if-then rules - specific to a given image based on the local information available - to be used by a fuzzy inference system. To this end, we only generate a partial histogram and not a complete histogram thus saving on computational costs. We show that the enhanced images from the proposed algorithm are either comparable, or in some cases, even better than those obtained from histogram equalization.

\section{Fuzzy Rule Based Inference System}

Fuzzy Inference Systems (FIS) are one of the best known applications of fuzzy set theory. They can be seen as a mapping between sets of fuzzy sets. Let $X, Y$ be non-empty sets and $f: X \rightarrow Y$ be any continuous function. Let $\mathbb{F}(X), \mathbb{F}(Y)$ denote the set of all fuzzy sets defined on $X, Y$, respectively, i.e., $\mathbb{F}(X)=\{\mathcal{A} \mid \mathcal{A}: X \rightarrow[0,1]\}$. Let an input $x \in X$ be given to the fuzzy inference system. With the help of a fuzzy if-then rule base and fuzzy logic operations on $[0,1]^{2}$, the inference module determines a corresponding output $y \in Y$. Thus, an FIS approximates $f$ by a fuzzy function $\tilde{f}: \mathbb{F}(X) \rightarrow \mathbb{F}(Y)$. The inference mechanism in an FIS itself can differ in their strategy of combining rules, choice of fuzzy logic operations, etc., and hence lead to various established types of FIS, viz., TS-fuzzy systems, Mamdani, Relational FIS, etc.

A fuzzy inference system can be completely and uniquely characterised by the following 6-tuple: $\mathcal{F}=\left(X, Y, \mathcal{A}_{i}, \mathcal{B}_{i}, \mathcal{R}, \mathcal{O}\right)$, where

- $X, Y$ are the input and output domains;

- $\mathcal{A}_{i}, \mathcal{B}_{i}$ are the fuzzy sets partitioning $X, Y$;

- $\mathcal{R}$ is a set of $n$ Single-Input Single-Output fuzzy rules of the form $(i=1, \ldots, n)$ : $R_{i}$ : If $\tilde{x}$ is $\mathcal{A}_{i}$ then $\tilde{y}$ is $\mathcal{B}_{i}$;

- $\mathcal{O}$ is the set of fuzzy logic operations used in the inference.

In the sequel, we describe only the Mamdani FIS in detail which is employed in this work.

Let us be given $n$ rules of the form "If $X$ is $\mathcal{A}_{i}$ then $Y$ is $\mathcal{B}_{i}$ ", where $\mathcal{A}_{i} \in \mathbb{F}(X), \mathcal{B}_{i} \in \mathbb{F}(Y)$ for $i=1, \ldots, n$. Let $x^{\prime} \in X$ be the given input. The appropriate output $y^{\prime} \in Y$ is determined in the Mamdani FIS by the following procedure:

Step 1 Calculate the degree of belongingness $s_{i}=$ $\mathcal{A}_{i}(x) \in[0,1]$.

Step 2 Obtain the modified output fuzzy set: $\mathcal{B}^{\prime}{ }_{i}(y)=s_{i} * \mathcal{B}_{i}(y)$, for all $y \in Y$.

Step 3 Obtain the overall modified output fuzzy set: $\mathcal{B}^{\prime}(y)=\mathcal{B}^{\prime}{ }_{1}(y) \circ \mathcal{B}^{\prime}{ }_{2}(y) \circ \ldots \circ \mathcal{B}^{\prime}{ }_{n}(y)$, for all $y \in Y$.

Step 4 Defuzzify the overall modified output fuzzy set: $y^{\prime}=\sum_{y \in Y} \frac{\mathcal{B}^{\prime}(y) \cdot y}{\mathcal{B}^{\prime}(y)}$.

The above Mamdani FIS can be characterised by $\mathcal{F}_{M}=\left(X, Y, \mathcal{A}_{i}, \mathcal{B}_{i}, \mathcal{R}, \mathcal{O}\right)$, where $\mathcal{O}=\left\{*=T_{\mathbf{M}}=\right.$ $\min , \cdot=T_{\mathbf{P}}=$ product, $\left.\circ=S_{\mathbf{M}}=\max \right\}$. 


\section{Fuzzy Techniques for Image Enhancement}

Let $\mathcal{I}$ denote an image with $M \times N$ pixels each with varying intensities in some subset of the range $[0, L-1]$ be given. Though, in the literature many works have appeared employing fuzzy techniques covering all aspects of image enhancement, viz., noise removal, sharpness enhancement, etc. (see for instance, $[4,17,18]$ ), we restrict ourselves only to those works dealing with contrast stretching or grey-level transformations.

The first such work was done by PAL and KING [14] wherein they propose a Contrast Intensification (CI) Operator, which was further generalised by DE and ChatterJi [5]. Unlike the approach taken in the present work, they consider the whole image $\mathcal{I}$ as a single fuzzy set $\mathcal{G}: X \longrightarrow[0,1]$, where $X$ is the set of all $M \times N$ pixels $x_{m n}$ and $\mathcal{G}\left(x_{m n}\right) \in$ $[0,1]$ gives the brightness of the corresponding pixel. Now they apply the $\mathbf{C I}$ operator, which is a function $T_{C I}:[0,1] \longrightarrow[0,1]$ on $\mathcal{G}\left(x_{m n}\right)$ and by taking the inverse of this composition the enhanced image is obtained.

Other fuzzy techniques used predominantly for contrast adaptation are (see [25] for more details):

- Minimization of fuzziness [5, 14, 15, 16, 25];

- Equalisation using fuzzy expected value: application of the most typical gray level [7, 19];

- Hyperbolization: nonlinear modification of membership values $[6,21]$;

- $\lambda$-enhancement: optimization of fuzziness using involutive membership functions [24];

- Enhancement using fuzzy relations [1].

We refer the readers to the following excellent monographs and surveys for more on fuzzy techniques for image processing: [16, 21, 25, 26].

\subsection{Fuzzy Rule Based Inference System for Contrast Enhancement}

Recall that a fuzzy inference system is completely and uniquely characterised by the following $\mathcal{F}=$ $\left(X, Y, \mathcal{A}_{i}, \mathcal{B}_{i}, \mathcal{R}, *, \circ\right)$ and that a fuzzy inference system can be thought of as a function approximator or a mapping from $X \rightarrow Y$.

It is clear now, that by taking $X \subset[0, L-1] ; Y=$ $[0, L-1]$ one can approximate the contrast stretching transformation $T: X \longrightarrow Y$ suitably by a fuzzy inference system $\mathcal{F}=(X \subset[0, L-1],[0, L-$ 1], $\left.\mathcal{A}_{i}, \mathcal{B}_{i}, \mathcal{R}, \mathcal{O}\right)$.

\subsubsection{Tizhoosh's Approach}

Fuzzy Rule-based approach is another technique employed and is the one taken in the current work. This approach has turned out to be both powerful and universal for many tasks in image processing. In Tizhoosh et al., [23] a simple inference system is proposed to increase the contrast of medical image data. Based on the full histogram of the image $\mathcal{I}$ they partition the input domain $X=[0, L-1]$ into 3 fuzzy sets, viz., dark, gray and bright. On the output domain they use 3 singleton fuzzy sets and obtain the input to output mapping as a weighted average of the membership values and these singletons.

\subsubsection{Proposed Approach}

To the best of the authors' knowledge, Tizhoosh's work is the only one to have taken this approach. Also, as mentioned in Tizhoosh et al., [23], their work was primarily intended to show the potential of fuzzy rule-based approach for image enhancement and was not further explored in a detailed manner. In this work, we intended to do this.

Towards this end, we consider here only fuzzy sets whose membership functions are triangular and employ the Mamdani fuzzy system. Thus, to approximate $T \sim \mathcal{F}$ we need to determine a fuzzy rule base or equivalently a fuzzy partition for the input and output domains. In this work, we propose a method of automatically generating an appropriate fuzzy rule base utilising the local image-specific information available, which when incorporated in to a Mamdani fuzzy inference system captures the above mapping.

\section{A Novel FIS for Contrast Enhancement}

Firstly, we define the following terms which will be necessary to understand the proposed algorithm.

Partial Histogram: In this work, for arriving at the fuzzy partition of the domains, instead of using the classical histogram we only use the partial histogram of the image, i.e., the histogram obtained by taking the pixel values only from some random paths in the pixel matrix. Typically, we have used 4 paths: 2 along the diagonals, 1 horizontal and 1 vertical. We find that the partial histogram still retains enough information about the distribution of pixel intensities for us to exploit it in the proposed algorithm.

Dynamic Minimum $D_{m}$ : Minimum value among all pixel intensities in the partial histogram.

Concentrated Minimum $C_{m}$ : Minimum value among all pixel intensities in the concentrated range of the partial histogram.

Concentrated Maximum $C_{M}$ : Maximum value among all pixel intensities in the concentrated range of the partial histogram.

Dynamic Maximum $D_{M}$ : Maximum value among all pixel intensities in the partial histogram. 
Please refer to Fig. 1(a) for a graphical explanation of the above parameters.

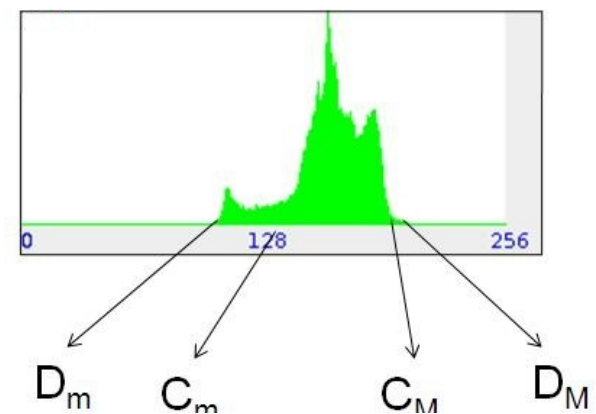

(a) Partial Histogram

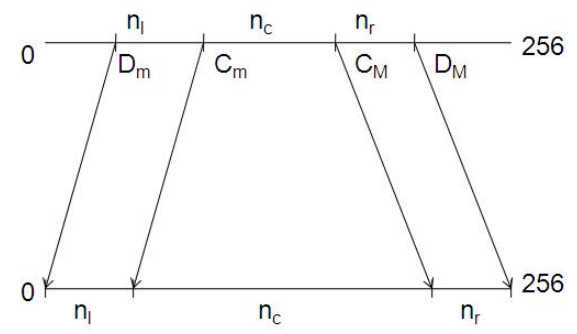

(b) Pictorial representation

Figure 1: (a) Partial Histogram with the Dynamic and Concentrated Minima \& Maxima; (b) Pictorial representation of step-3 of the proposed algorithm (see Section 4.1)

Note that since the pixel intensities vary only between $\left[D_{m}, D_{M}\right]$ this interval becomes the de facto input domain $X$. Let us denote by $\mathcal{F}_{C S}$ the Mamdani fuzzy inference system characterized by $\mathcal{F}_{C S}=\left(\left[D_{m}, D_{M}\right],[0, L-\right.$ 1], $\mathcal{A}_{i}, \mathcal{B}_{i}, \mathcal{R},\{\min$, product, $\left.\max \}\right)$. The following algorithm specifies how to determine the fuzzy partitions $\mathcal{A}_{i}, \mathcal{B}_{i}$.

\subsection{The Proposed Algorithm}

Step 1 From the image $\mathcal{I}$ plot the partial histogram by choosing a few random paths and hence determine the 4 parameters: $D_{m}, C_{m}, C_{M}, D_{M}$.

Step 2 Partition the sub-domains $\left[D_{m}, C_{m}\right],\left[C_{m}+\right.$ 1, $\left.C_{M}\right],\left[C_{M}+1, D_{M}\right]$ of the input domain $X=$ $[0, L-1]$ into $n_{l}, n_{c}, n_{r}$ number of fuzzy sets, respectively. Thus there are $n=n_{l}+n_{c}+n_{r}$ input fuzzy sets $\mathcal{A}_{i}, i=1,2, \ldots, n$. Note that each of the $\mathcal{A}_{i}$ 's is a triangular fuzzy set and hence requires only the 3 parameters, viz., left, center and right points, to be specified. This is done as follows:

a) We choose $n_{l}$ equispaced points, say of step size $h_{l}$, in the sub-domain $\left[D_{m}, C_{m}\right]$ and for the triangular fuzzy set $\mathcal{A}_{i}, i=$ $1, \ldots, n_{l}$, the three parameters are specified as $\left(x_{l}, x_{c}, x_{r}\right)=\left(D_{m}+(i-1) \cdot h_{l}, D_{m}+\right.$ $\left.i . h_{l}, D_{m}+(i+1) \cdot h_{l}\right)$. b) Similarly, we choose $n_{c}, n_{r}$ equispaced points with step sizes $h_{c}, h_{r}$ on the other 2 sub-domains and assign them similarly as above, viz., the triangular fuzzy sets $\mathcal{A}_{i}, i=n_{l}+1, \ldots, n_{l}+n_{c}$, the three parameters are specified as $\left(x_{l}, x_{c}, x_{r}\right)=$ $\left(C_{m}+\left(i-n_{l}-1\right) \cdot h_{c}, C_{m}+\left(i-n_{l}\right) \cdot h_{c}, D_{m}+\right.$ $\left.\left(i-n_{l}+1\right) \cdot h_{c}\right)$, while the triangular fuzzy sets $\mathcal{A}_{i}, i=n_{l}+n_{c}+1, \ldots, n$, the three parameters are specified as $\left(x_{l}, x_{c}, x_{r}\right)=$ $\left(C_{M}+\left(i-n_{l}-n_{c}-1\right) \cdot h_{r}, C_{m}+\left(i-n_{l}-\right.\right.$ $\left.\left.n_{c}\right) \cdot h_{r}, D_{m}+\left(i-n_{l}-n_{c}+1\right) \cdot h_{r}\right)$.

Step 3 Similarly, partition the output domain $(Y=[0, L-1])$ into a total of $n$ fuzzy sets $\mathcal{B}_{i}$. This fuzzy partition is not done arbitrarily, but in such a way that the following holds:

(i) $D_{m} \in X$ is mapped to $0 \in Y$.

(ii) $D_{M} \in X$ is mapped to $255 \in Y$.

(iii) Pixels in the range of $\left(D_{m}, C_{m}\right]$, i.e., $x \in\left(D_{m}, C_{m}\right] \subset X$ are mapped to the $\left(0, C_{m}-D_{m}\right] \subset Y$.

(iv) Pixels in the range of $\left(C_{m}, C_{M}\right)$, i.e., $x \in$ $\left(C_{m}, C_{M}\right) \subset X$ are mapped to the range $\left(C_{m}-D_{m}, 255-D_{M}-C_{M}\right) \subset Y$.

(v) Pixels in the range of $\left[C_{M}, D_{M}\right)$, i.e., $x \in$ $\left[C_{M}, D_{M}\right) \subset X$ are mapped to the range $\left[255-D_{M}-C_{M}, 255\right) \subset Y$.

Since we use a Mamdani inference system, the above can be achieved by creating the output fuzzy set $\mathcal{B}_{i}$, where $i=1,2, \ldots, n$, as done above, i.e., we choose $n_{l}, n_{c}, n_{r}$ equispaced points, say of step size $n_{l}, n_{c}, n_{r}$, in the subdomains $\left[0, C_{m}-D_{m}\right],\left[C_{m}-D_{m}, 256-D_{M}-\right.$ $\left.C_{M}\right],\left[256-D_{M}-C_{M}, 256\right]$ and specify the 3 parameters $\left(x_{l}, x_{c}, x_{r}\right)$ as above. Note that the concentrated domain $\left(C_{m}, C_{M}\right)$ is mapped to a larger subset of $[0, L-1]$, viz., $\left[C_{m}-D_{m}, 256-\right.$ $\left.D_{M}-C_{M}\right]$ and hence, even though the number of equispaced points remains the same, viz., $n_{c}$, the step size $k_{c} \neq n_{c}$.

Step 4 Form the fuzzy if-then rule base consisting of $n$ rules by relating the $i$-th input fuzzy set $\mathcal{A}_{i}$ to the $i$-th output fuzzy set $\mathcal{B}_{i}$, where $i=$ $1,2, \ldots, n$.

Step 5 Employ the fuzzy system $\mathcal{F}_{C S}$ on the input sub-domain $X^{\prime}=\left[D_{m}, D_{M}\right]$ to get the contrast stretching transformation $T$.

\subsection{Comparison of the Proposed Approach with Histogram Equalization and Tizhoosh's Approach}

We applied the above algorithm employing a Mamdani fuzzy system with Triangular fuzzy sets for both the input and output fuzzy sets. The graylevel images were so chosen to cover a broad category of images, viz., outdoor -Boat image, indoor 


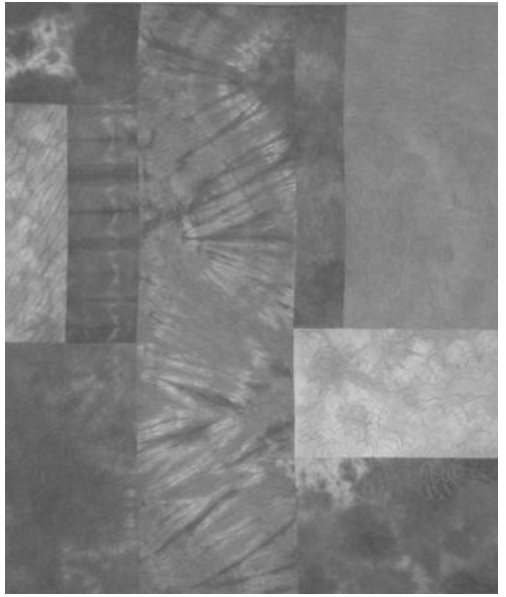

(a) Original image

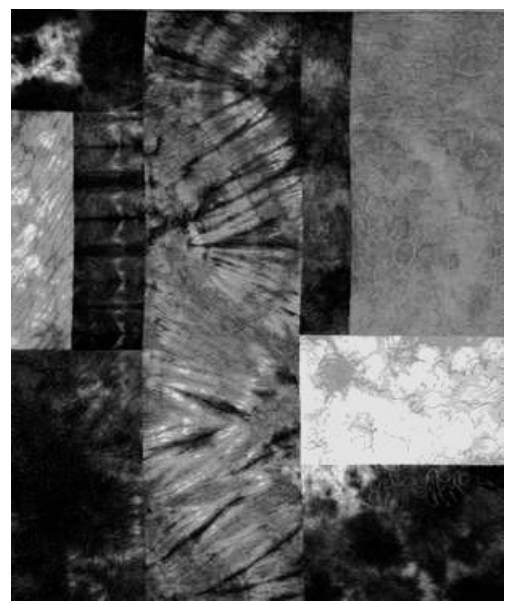

(c) Fuzzy inferred image

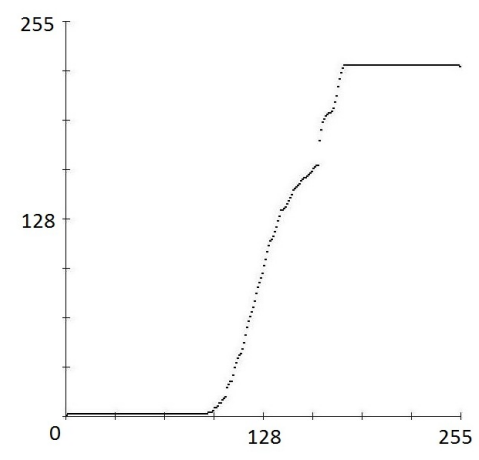

(d) Transformation Function

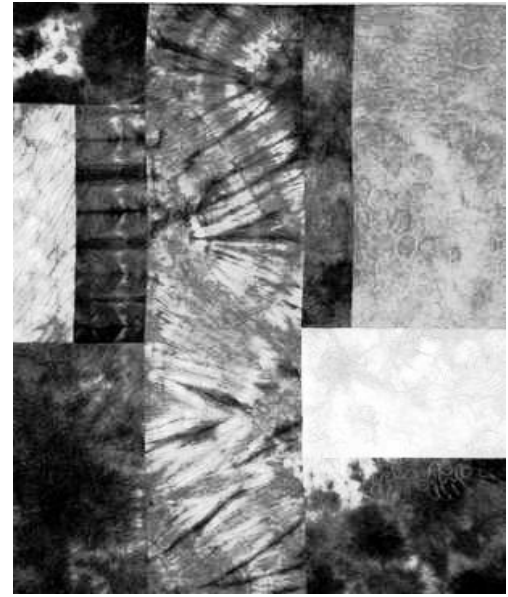

(b) Histogram equalized image

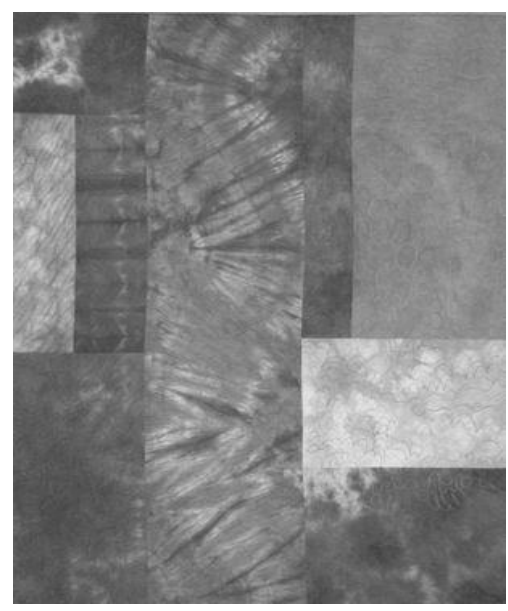

(e) Tizhoosh Approach

Figure 2: Results for the Design Pattern image

- Magazine cover and Design Pattern images and medical - X-ray image. In Figures. 2-5, we present the original image and also the enhanced images using histogram equalization, Tizhoosh's approach and the proposed approach in this work. We also give the final gray-scale transformation functions obtained. It can be observed that we obtain consistently good enhanced images from the proposed method.

\subsection{Advantages of the Proposed Method}

- Since we only require and compute the partial histogram instead of the complete histogram to determine the fuzzy partitioning of the input domain, the proposed method computationally compares favourably with that proposed in Tizhoosh et al., [23].

- The input and output fuzzy partitioning is done in a transparent and systematic way. Instead of using a priori transformations like Log, Inverse log transformations which usually do not exploit the image specific information available, the proposed method does exploit the image specific information available. Further, since fuzzy inference systems are known universal approximators (see TIKK et al. [20]) it is clear that by suitably partitioning the input and output domains with fuzzy sets one can obtain any of the classical a priori transformations to arbitrary accuracy.

- Note that even when the dynamic range of the given image is only a subset of $[0, L-1]$, the dynamic range of the enhanced image is whole of $[0, L-1]$ in the proposed method while that of histogram equalisation is at best $\left[D_{m}, L-1\right]$, and hence the proposed method is more likely to give better contrast to the resulting image.

- Note that histogram equalization is optimised to exploit the human visual range and hence is not always suitable if the contrast stretching stage is only a pre-processor for a subsequent machine processing stage. In contrast, the fuzzy system based gray level transformations can be tailor-made by tuning the fuzzy partitions of the domains.

- Finally, though we have considered only contrast stretching through piecewise-linear transforma- 


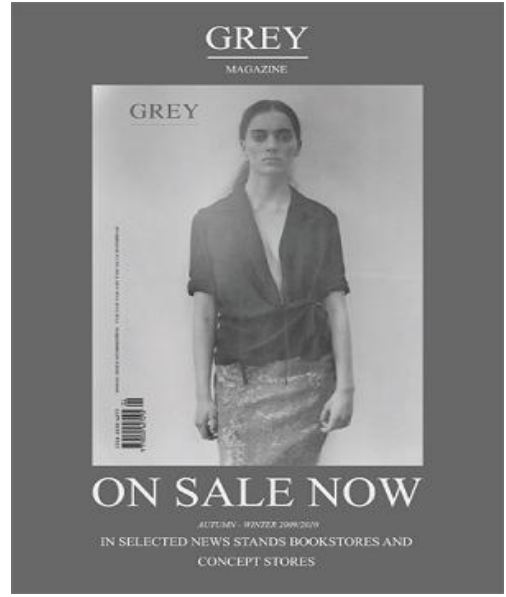

(a) Original image

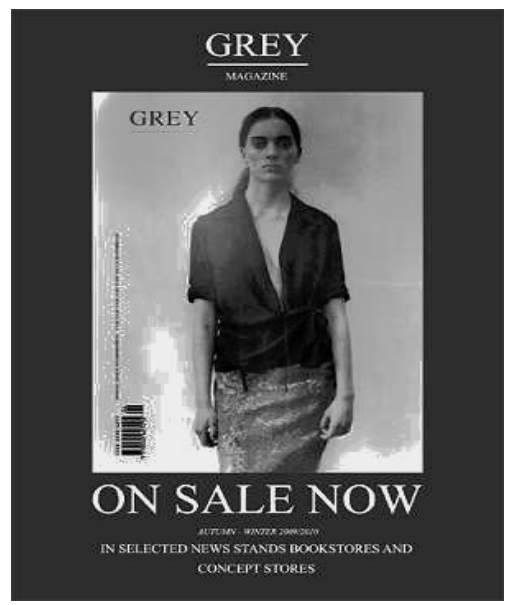

(c) Fuzzy inferred image

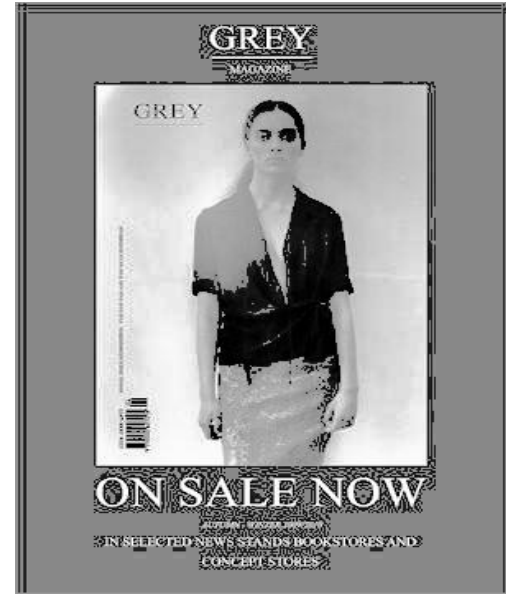

(b) Histogram equalized image

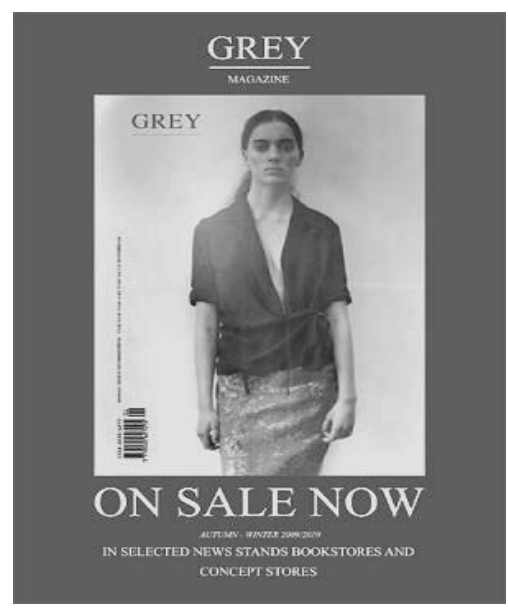

(e) Tizhoosh Approach

Figure 3: Results for Magazine Cover image

tions, the above system can be easily tuned, for instance by employing fuzzy sets of other shapes, to simulate any gray-level transformation function.

\subsection{Some concerns of the Proposed Method}

Despite the many advantages listed above, some concerns do remain and have been listed below.

- The current method is optimised for low-contrast images, i.e., those with unimodal histograms. Though this algorithm can be easily extended for images with multi-modal histograms by having multiple concentrated minima \& maxima and running the current algorithm on these many sub-domains, this method is plagued with the same concerns as that of local histogram method with non-overlapping neighbourhoods, viz., imbalance in overall contrast even though different parts of the image are well contrasted.

- The advantage of obtaining the required information from the partial histogram can be lost if the given image is not uniformly varying in contrast. In which case, one may have to resort to more number of random paths and in the extreme case requiring the whole histogram to decide on the partition.

\section{Conclusions}

In this work, we have proposed a fuzzy inference system based contrast enhancement of gray level images. The proposed method of generating the fuzzy if-then rules, which is specific to a given image based on the local information available, is obtained from the partial histogram and not a complete histogram thus saving on computational costs. We show that the enhanced images from the proposed algorithm are either comparable, or in some cases, even better than those obtained from histogram equalization.

A straightforward extension of this work is to automate the determination of fuzzy partitions that perform the different a priori transformations thus showing the versatility of the proposed framework. We would also would like to address the above listed concerns and work on porting the above framework to colour images. 


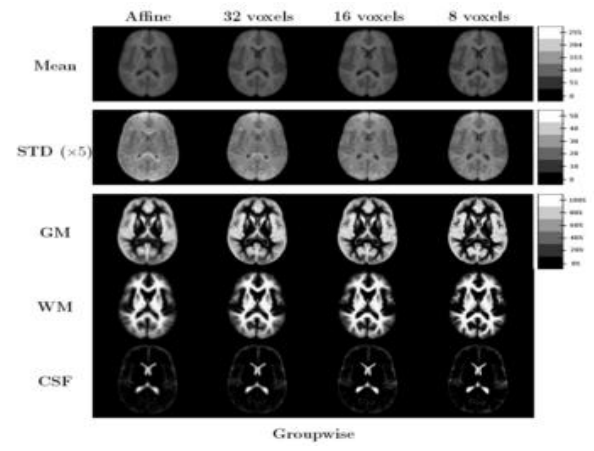

(a) Original image

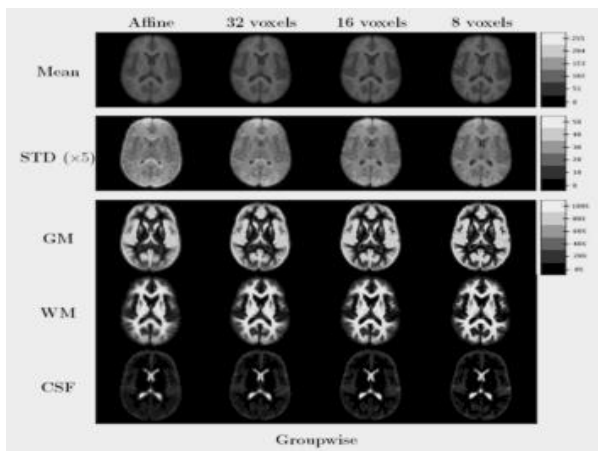

(c) Fuzzy inferred image

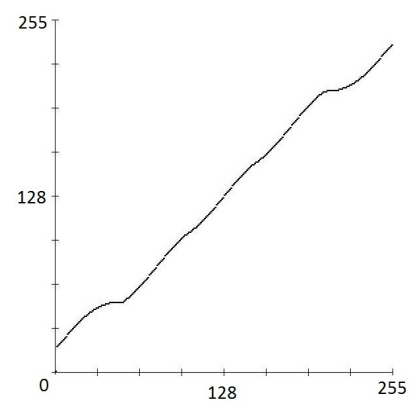

(d) Transformation Function

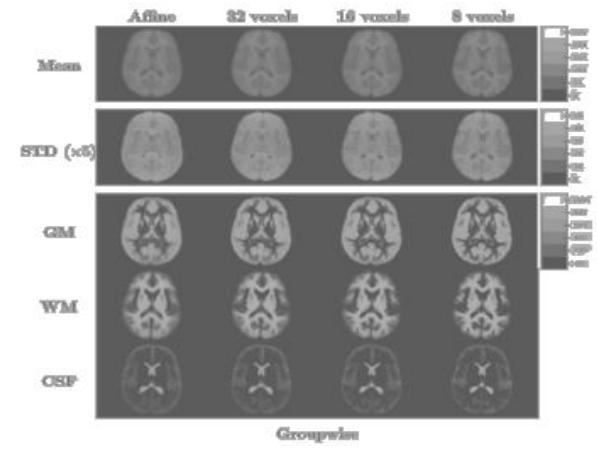

(b) Histogram equalized image

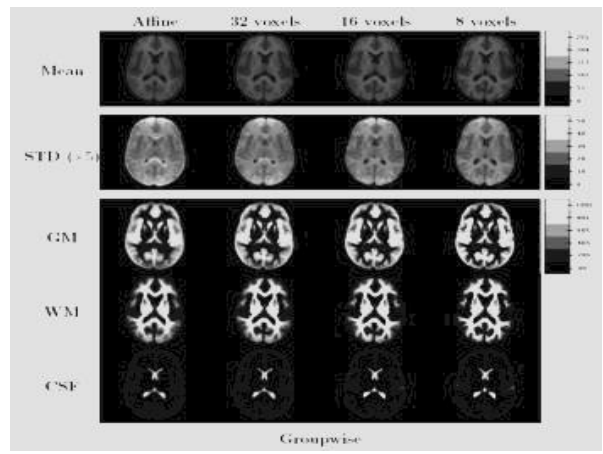

(e) Tizhoosh Approach

Figure 4: Results for the X-ray image-Note the enhancement attained for the subimages in the last row of (c)

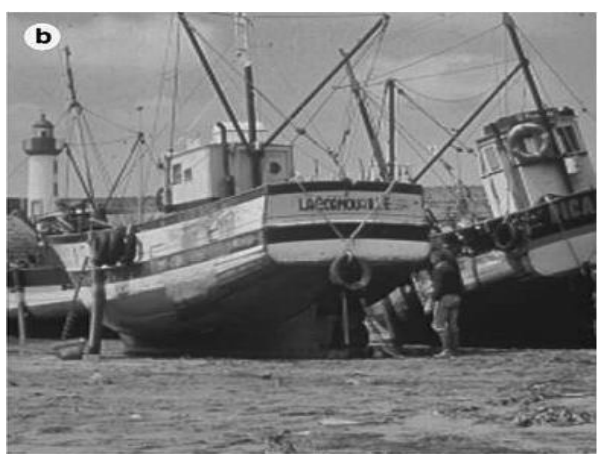

(a) Original image

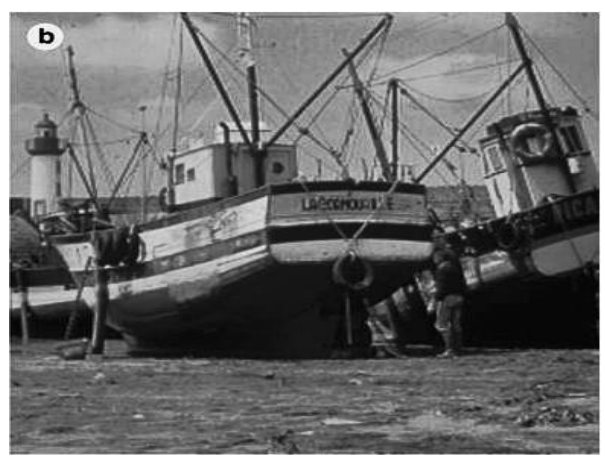

(c) Fuzzy inferred image

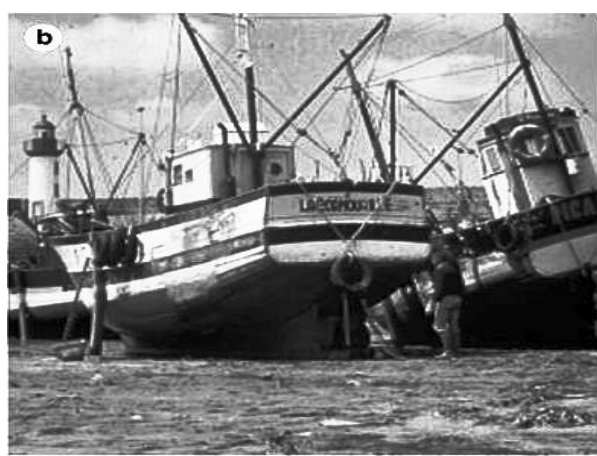

(b) Histogram equalized image

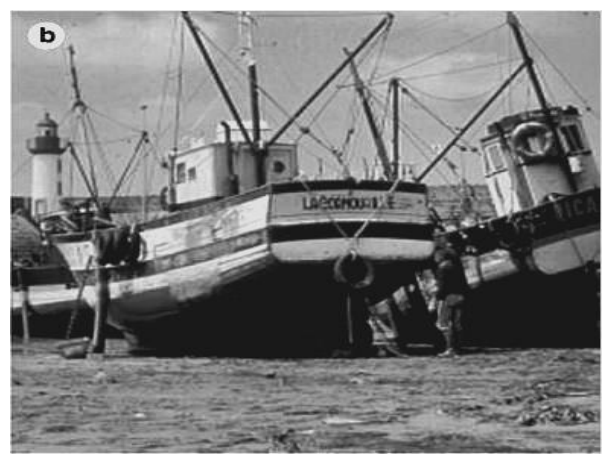

(e) Tizhoosh Approach

Figure 5: Results for the Boat image 


\section{References}

[1] Bhutani K.R., Battou A. An application of fuzzy relations to image enhancement. Pattern Recognition Letters, 16: 901-909, 1995.

[2] Chen B.-T, Chen Y.-S, Hsu W.-H. Automatic histogram specification based on fuzzy set operations for image enhancement. IEEE Signal Processing Letters, 2(2): 37-40, 1995.

[3] Chen B.-T, Chen Y.-S, Hsu W.-H. Image Processing and understanding based on fuzzy inference approach. In Proc. FUZZ-IEEE'94, Vol I: 254-259, Orlando, FL, USA, June, 1994.

[4] Choi Y.S., Krishnapuram R. A robust approach to image enhancement based on fuzzy logic. IEEE Trans. on Image Processing, 6(6): 808-825, 1997.

[5] De T.K., Chatterji B.N. An approach to a generalised technique for image contrast enhancement using the concept of fuzzy set. Fuzzy Sets and Systems 25(2):145-158, 1988.

[6] Frei W. Image enhancement by histogram hyperbolization. In CGIP 6(3): 397-406, 1977.

[7] M. Friedman, M. Schneider, A. Kandel. The use of weighted fuzzy expected value (WFEV) in fuzzy expert systems. Fuzzy Sets and Systems 31(1):37-45, 1989.

[8] Gonzalez R.C., Woods R. E. Digital Image Processing, 3rd Ed. Prentice Hall Inc., 2008.

[9] Gottwald S. (2001) A treatise on many-valued logics. Research Studies Press, Baldock, Hertfordshire.

[10] E. E. Kerre and M. Nachtegael (Ed.) (2000) Fuzzy Techniques in Image Processing. Physica-Verlag, Heidelberg.

[11] Klement E.P., Mesiar R., Pap E. (2000) Triangular norms. Kluwer, Dordrecht.

[12] Klir G.J., Yuan Bo (1995) Fuzzy sets and fuzzy logic. Theory and applications. Prentice Hall, New Jersey.

[13] Menger K. (1942) Statistical metrics. Proc. Nat. Acad. Sci. USA. 28:535-537.

[14] Pal S.K., King R.A. Image enhancement using smoothing with fuzzy sets. IEEE Trans. on Syst. Man and Cybern., 11(7): 494-501, 1981.

[15] Pal S.K., Rosenfeld A. Image enhancement and thresholding by optimization of fuzzy compactness. Pattern Recognition Letters, 7: 77-86, 1988.

[16] Pal S.K., Dutta Majumdar D. Fuzzy Mathematical Approach to Pattern Recognition. John Wiley and Sons, New York, 1986.

[17] Russo F., Ramponi G. A fuzzy filter for images corrupted by impulse noise. IEEE Signal Processing Letters, 3(6): 168-170, 1996.

[18] Russo F. FIRE operators for image processing. Fuzzy Sets and Systems 103:265-275, 1999.

[19] M. Schneider, M. Craig On the use of fuzzy sets in histogram equalization. Fuzzy Sets Syst. 45(3):271-278, 1992.
[20] Tikk D., Kóczy L.T., Gedeon T.D. A survey on universal approximation and its limits in soft computing techniques. Int. Jl. of Approx. Reas. 33(2):185-202, 2003.

[21] Tizhoosh, H.R. Fuzzy Image Processing (in German). Springer, 1997.

[22] Tizhoosh, H.R., Krell G., Michaelis B. Locally Adaptive Fuzzy Image Enhancement. In B. Reusch (Ed.), Computational Intelligence, Theory and Applications, Proc. Of 5th Fuzzy Days'97, 272-276, Dortmund, Germany, Springer, 1997.

[23] Tizhoosh, H.R., Krell, G., Michaelis, B. On Fuzzy Image Enhancement of Megavoltage Images in Radiation Therapy. In FUZZ-IEEE'97, 1399-1404, Spain, 1997.

[24] Tizhoosh, H.R., Krell, G., Michaelis, B. Lambda-Enhancement: Contrast adaptation based on Optimization of Image Fuzziness. In FUZZ-IEEE'98, 1548-1553, Alaska, USA, 1998.

[25] Tizhoosh, H.R. Fuzzy Image Enhancement: An Overview. In Kerre, E., Nachtegael, M. (Eds.): Fuzzy Techniques in Image Processing, Springer, Studies in Fuzziness and Soft Computing, pp. 137-171, 2000.

[26] Tizhoosh, H.R., Hauschecker, H. Fuzzy Image Processing: An Overview. In Jähne, B., Hauschecker, H. (Eds.), Computer vision and applications: A Guide for Students and Practitioners, Academic Press, Boston, 2000, pp. 541-576, 